\title{
APPLYING THE MOUNTAIN PASS THEOREM TO AN ASYMPTOTICALLY LINEAR ELLIPTIC EQUATION ON $\mathbb{R}^{N}$
}

\author{
C.A. Stuart \\ Department of Mathematics, \\ Ecole Polytechnique Fédérale de Lausanne, \\ CH-1015 Lausanne, Switzerland
}

\section{H.S. Zhou}

Wuhan Institute of Physics and Mathematics, Academia Sinica, P.O.Box 71010, Wuhan 430071, P.R. China

\section{Introduction}

We consider the nonlinear elliptic eigenvalue problem:

$$
\left\{\begin{array}{l}
-\triangle u(x)=\lambda u(x)+f(|x|, u(x)) u(x), \quad \text { for } \quad x \in \mathbb{R}^{N} \\
u \in H^{1}\left(\mathbb{R}^{N}\right), \quad u \neq \equiv 0
\end{array}\right.
$$

where $N \geq 3, \triangle$ denotes the $N$-dimensional Laplacian and the function $f$ satisfies the following conditions:

(H1) $f \in C\left([0, \infty)^{2}\right)$ and $\lim _{s \rightarrow 0} f(r, s)=0$ uniformly for $r \geq 0$.

(H2) For all $r \geq 0, f(r, s)$ is a non-decreasing function of $s$ on $[0, \infty)$ and there exists $g(r)<\infty$ such that

$$
\lim _{s \rightarrow \infty} f(r, s)=g(r) \quad \text { uniformly for } \quad r \geq 0 .
$$

Furthermore,

$$
\lim _{r \rightarrow \infty} g(r)=g(\infty) \quad \text { exists and } \quad 0<g(\infty)<\infty .
$$


Remark 1.1: Clearly $g \in C([0, \infty)) \cap L^{\infty}(0, \infty)$ and $0 \leq f(r, s) \leq g(r)<$ $\infty$, for all $r, s \geq 0$.\#

For a function $f$ satisfying $(H 1)$ we set

$$
F(r, s)=\int_{0}^{s} f(r, t) t d t \quad \text { for } \quad r, s \geq 0 .
$$

Remark 1.2: For any function $f$ satisfying $(H 1)$ and $(H 2)$, we have

$$
0 \leq F(r, s) \leq \frac{1}{2} g(r) s^{2} \quad \text { for all } \quad r, s \geq 0
$$

Also there is $R>0$ such that $\inf _{r \geq R} g(r)>0$ and $0<\lim _{s \rightarrow+\infty} \frac{F(r, s)}{s^{2}}<\infty$ uniformly for $r \geq R$. This means that there is no number $\theta>0$ such that

$$
(2+\theta) F(r, s) \leq f(r, s) s^{2} \quad \text { for all } \quad s \geq 0, r \geq R
$$

since this inequality implies that $s^{-(2+\theta)} F(r, s)$ is non-decreasing in $s$, and consequently that $\lim _{s \rightarrow \infty} s^{-\theta} f(r, s)>0$, contradicting the boundedness of $f(r, s)$ as $s \rightarrow \infty$.\#

Under the hypotheses $(H 1)$ and $(H 2)$, we obtain the following results concerning the problem (1.1). We use the standard notation for function spaces with $H^{k}\left(\mathbb{R}^{N}\right)=W^{k, 2}\left(\mathbb{R}^{N}\right)$ for $k=1,2$. The norm of an element $u \in L^{p}\left(\mathbb{R}^{N}\right)$ will be denoted by $|u|_{p}$.

Definition: Consider a function $f$ satisfying $(H 1)$ and $(H 2)$. By a solution of the problem (1.1) we mean a pair $(\lambda, u) \in R \times H^{1}\left(\mathbb{R}^{N}\right)$ such that $u(x) \geq 0$ a.e. on $\mathbb{R}^{N}, u(x)>0$ for $x$ in a set of positive measure and

$$
\int_{\mathbb{R}^{N}} \nabla u(x) \cdot \nabla v(x) d x=\int_{\mathbb{R}^{N}}\{\lambda+f(|x|, u(x))\} u(x) v(x) d x
$$

for all $v \in C_{0}^{\infty}\left(\mathbb{R}^{N}\right)$. Noting that $(H 1)$ and $(H 2)$ imply that $x \longmapsto f(x, u(|x|))$ is in $L^{\infty}\left(\mathbb{R}^{N}\right)$ for every non-negative measurable function $u$ on $\mathbb{R}^{N}$, we see that (1.4) is equivalent to the same identity for all $v \in H^{1}\left(\mathbb{R}^{N}\right)$.

In Section 2, we show that for every solution $(\lambda, u)$ of $(1.1)$, the function $u$ is continuous on $\mathbb{R}^{N}$ with $u(x)>0$ for every $x \in \mathbb{R}^{N}$ and $\lim _{|x| \rightarrow \infty} u(x)=0$. Our main results concern the existence of solutions of (1.1) and they can be 
summarized briefly as follows.

There is a number $\Lambda<0$ such that :

(i) if $(\lambda, u)$ is a solution of $(1.1)$, then $\lambda \in(\Lambda, 0]$, and

(ii) for every $\lambda \in(\Lambda, 0)$ there exists a solution $(\lambda, u)$ of $(1.1)$ where $u$ is strictly positive and radially symmetric.

We show by examples in Section 2 that the existence or not of solutions of (1.1) with $\lambda=0$ cannot be determined from the hypotheses $(H 1)$ to $(H 2)$.

In these results the number $\Lambda$ is defined by

$\Lambda=\inf \left\{\int_{\mathbb{R}^{N}}|\nabla u(x)|^{2}-g(|x|) u(x)^{2} d x: u \in H^{1}\left(\mathbb{R}^{N}\right)\right.$ and $\left.\quad \int_{\mathbb{R}^{N}} u(x)^{2} d x=1\right\}$.

Clearly, $\Lambda \geq-|g|_{\infty}>-\infty$ and, as is well-known [2], $\Lambda=\inf \sigma(S)$ where $\sigma(S)$ denotes the spectrum of the self-adjoint operator $S: \mathcal{D}(S) \subset L^{2}\left(\mathbb{R}^{N}\right) \longrightarrow$ $L^{2}\left(R^{N}\right)$ defined by

$$
\mathcal{D}(S)=H^{2}\left(\mathbb{R}^{N}\right) \text { and } S u(x)=-\triangle u(x)-g(|x|) u(x) \text { for } u \in H^{2}\left(\mathbb{R}^{N}\right) .
$$

Furthermore, the essential spectrum of $S$ is the interval $[-g(\infty), \infty)$ and so we have that

$$
-|g|_{\infty} \leq \Lambda \leq-g(\infty)<0 .
$$

The operator $S$ plays a crucial role since it defines the asymptotic linearization of (1.1). The corresponding operator for the linearization of (1.1) at $u \equiv 0$ is defined by $\mathcal{D}(L)=H^{2}\left(\mathbb{R}^{N}\right)$ and $L u(x)=-\triangle u(x)$ for $u \in H^{2}\left(\mathbb{R}^{N}\right)$. Here $\sigma(L)=[0, \infty)$ and the fundamental interval $(\Lambda, 0)$ in our results should be seen as (inf $\sigma(S)$, inf $\sigma(L)$ ). For $\lambda \in \sigma(S)$ the problem (1.1) is said to be in resonance and this can occur either because $\lambda$ is an eigenvalue of $S$ or because $\lambda$ lies in the essential spectrum, $\sigma_{e}(S)$, of $S$. Furthermore, $[-g(\infty), 0)$ is nonempty and is contained in $\sigma_{e}(S)$, so the problem (1.1) is always in resonance for infinitely many points in $(\Lambda, 0)$. On the other hand, $(\Lambda,-g(\infty)) \cap \sigma_{e}(S)=\emptyset$ and all points in $(\Lambda,-g(\infty))$ (which may be empty) are either regular values or eigenvalues of $S$. The existence of a solution to (1.1) is established for every $\lambda \in(\Lambda, 0)$ irrespective of whether or not the problem is in resonance. However, in several places, different proofs have to be given depending on which of the following cases occurs :

Case $1 \quad \Lambda<-g(\infty) \quad$ or Case $2 \quad \Lambda=-g(\infty)$.

In Section 3, our proof of the existence of solutions is based on a variant of 
the mountain pass theorem [1], which we state as Proposition 3.4 in Section 3 for the reader's convenience. For all $\lambda<0$, the underlying functional $J$ has the appropriate behaviour near 0 but, since (1.1) is asymptotically linear near infinity, the functional $J$ has an indefinite character near infinity for $\lambda>\Lambda$. This means that the usual method [7] for obtaining a bounded Palais-Smale sequence for $J$, which is based on an inequality like (1.3), cannot be used. In using the result of Bartolo, Benci and Fortunato, [1], to deal with this problem the main difficulties are in showing that the conditions (b) and (d) of Proposition 3.4 are satisfied. A first result in this direction is given in [11]. In the present approach, which yields a more general result, our method of establishing the property (d) was inspired by a recent paper [6] by L. Jeanjean where a problem with similar features is treated by another variant of the mountain pass method. His approach to establishing the boundedness of a P-S sequence lies at the heart of our method of proving the condition $(d)$.

Our results concerning the regularity and non-existence of solutions of (1.1) are given in Section 2. Before turning to this, let us observe a simple, but useful, way of constructing functions $f$ which satisfy the conditions $(H 1)$ and $(H 2)$. It will be used again in Section 2 to find a class of functions $f$, satisfying $(H 1)$ and $(H 2)$, for which (1.1) has no solutions with $\lambda=0$ and another class for which (1.1) has a radially symmetric solution with $\lambda=0$.

Suppose that

(A) $q \in C([0, \infty))$ with $q(r) \geq 0$ for all $r \geq 0$. Also, $Q \equiv \lim _{r \rightarrow \infty} q(r)$ exists and $0<Q<\infty$.

(B) $p \in C^{1}((0, \infty))$ with $p^{\prime}(s) \geq 0$ for all $s>0$ and $0=\lim _{s \rightarrow 0} p(s)<P \equiv$ $\lim _{s \rightarrow \infty} p(s)<\infty$. We extend $p$ continuously by setting $p(0)=0$.

Remark 1.4: Let $f$ be a function satisfying $(H 1)$ and $(H 2)$ and let $q$ satisfy $(A)$. Then the function $h$ defined by $h(r, s)=q(r) f(r, s)$ also satisfies the conditions $(H 1)$ and $(H 2)$.\#

Remark 1.5: Let $q$ and $p$ be functions satisfying the conditions $(A)$ and $(B)$ respectively. Then, it is easy to see that the function $f$ defined by

$$
f(r, s)=\left\{\begin{array}{lllll}
q(r) p(s) & \text { for } & r \geq 0 & \text { and } & s>0 \\
0 & \text { for } & r \geq 0 & \text { and } & s=0
\end{array}\right.
$$

satisfies the conditions $(H 1)$ and $(H 2)$.\# 


\section{Regularity and non-existence of solutions}

We begin by showing that for all solutions $(\lambda, u)$ of $(1.1)$, the function $u$ has additional smoothness and is strictly positive on $\mathbb{R}^{N}$. This discussion is based on the following properties of the Laplacian. Recall that if $u \in L^{p}\left(\mathbb{R}^{N}\right)$ for some $p$ with $1 \leq p \leq \infty$, then $u$ defines a tempered distribution.

Proposition 2.1 (a) For each tempered distribution $h$ on $\mathbb{R}^{N}$, there exists a unique tempered distribution $u=T h$ satisfying the equation $-\triangle u+u=h$ on $\mathbb{R}^{N}$ in the sense of distributions.

(b) Let $h \in L^{p}\left(\mathbb{R}^{N}\right)$ for some $p$ with $1 \leq p \leq \infty$. Then, $T h \in L^{p}\left(\mathbb{R}^{N}\right)$ and $|T h|_{p} \leq|h|_{p}$. In the case where $p \in(1, \infty)$, we even have $T h \in W^{2, p}\left(\mathbb{R}^{N}\right)$ and there is a constant $C(p, N)$ such that

$$
\|T h\|_{W^{2, p}\left(\mathbb{R}^{N}\right)} \leq C(p, N)|h|_{p} .
$$

Proof: This follows from Proposition 27 in Chapter II $\S 8$ of [3] combined with the Calderon-Zygmund estimate $\left|\partial_{i} \partial_{j} u\right|_{p} \leq A(p, N)|\triangle u|_{p}$ (Proposition 3 in Chapter 3 of [8]).\#

Theorem 2.2 Let $f$ be a function satisfying the conditions $(H 1)$ and $(H 2)$. Let $(\lambda, u)$ be a solution of (1.1). Then

(i) $u \in W^{2, p}\left(\mathbb{R}^{N}\right)$ for all $p \in[2, \infty)$ and

$$
-\triangle u(x)=\lambda u(x)+f(|x|, u(x)) u(x) \quad \text { a.e. on } \quad \mathbb{R}^{N} \text {. }
$$

(ii) $u \in C^{1}\left(\mathbb{R}^{N}\right), \lim _{|x| \rightarrow \infty} u(x)=0$ and $\lim _{|x| \rightarrow \infty} \nabla u(x)=0$.

(iii) $u(x)>0$ for all $x \in \mathbb{R}^{N}$.

Proof: Since $0 \leq f(r, s) \leq g(r) \leq|g|_{\infty}<\infty$ for all $r, s \geq 0$, we have that

$$
|\{\lambda+1+f(|x|, u(x))\} u(x)|_{p} \leq\left\{|\lambda+1|+|g|_{\infty}\right\}|u|_{p},
$$

for all $p \in[1, \infty)$. Setting

$$
h(x)=\{\lambda+1+f(|x|, u(x))\} u(x) \text { on } \quad \mathbb{R}^{N} .
$$

We see that $h \in L^{p}\left(\mathbb{R}^{N}\right)$ for $2 \leq p \leq \frac{2 N}{N-2}$ and $u \in H^{1}\left(\mathbb{R}^{N}\right)$ defines a tempered distribution satisfying

$$
-\triangle u(x)+u(x)=h(x) \quad \text { in } \quad \mathbb{R}^{N}
$$


in the sense of distributions. By Proposition 2.1(a), $u=T h$ and so $u \in$ $W^{2, p}\left(\mathbb{R}^{N}\right)$ for $2 \leq p \leq \frac{2 N}{N-2}$ by Proposition $2.1(\mathrm{~b})$. Hence $u$, and consequently $h$, belongs to $L^{p}\left(\mathbb{R}^{N}\right)$ for $2 \leq p<\infty$ if $N \leq 6$ and $2 \leq p \leq \frac{2 N}{N-6}$ if $N>6$. A standard "boot-strap" argument now completes the proof of part (i).

The properties in part (ii) hold for any element $u$ of $W^{2, p}\left(\mathbb{R}^{N}\right)$ provided that $p>N$. Since $u(x) \geq 0$ a.e. on $\mathbb{R}^{N}$ for any solution of (1.1),

$$
-\triangle u(x)+|\lambda| u(x)=\{\lambda+|\lambda|+f(|x|, u(x))\} u(x) \geq 0 \quad \text { a.e. on } \quad \mathbb{R}^{N}
$$

and so the strong maximum principle (Theorem 8.19 of [5]) shows that $u(x)>0$ for all $x \in \mathbb{R}^{N}$. This proves part (iii).\#

Theorem 2.3 Let $f$ be a function satisfying the conditions $(H 1)$ and $(H 2)$ and let $(\lambda, u)$ be a solution of (1.1). Then $\Lambda<\lambda \leq 0$.

Proof: For $p>0$, let $J_{p}$ denote the Bessel function of the first kind of order $p$ and let $\alpha_{p}>0$ be the smallest postive zero of $J_{p}$.

For $k>0$, we set

$$
w_{k}(x)=\left\{\begin{array}{lll}
|k x|^{\frac{2-N}{2}} J_{\frac{N-2}{2}}(k x) & \text { for } & x \in \mathbb{R}^{N} \backslash\{0\} \\
\frac{1}{2^{\frac{N-2}{2}} \Gamma\left(\frac{N}{2}\right)} & \text { for } & x=0 .
\end{array}\right.
$$

Then, as is well-known [4], $w_{k} \in C^{\infty}\left(\mathbb{R}^{N}\right)$ and,

$$
\begin{gathered}
\triangle w_{k}(x)+k^{2} w_{k}(x)=0 \text { for all } x \in \mathbb{R}^{N}, \\
w_{k}(x)>0 \text { for }|x|<R(k, N), \\
w_{k}(x)=0>\frac{\partial}{\partial r} w_{k}(x) \text { for }|x|=R(k, N),
\end{gathered}
$$

where $R(k, N)=k^{-1} \alpha_{\frac{N-2}{2}}$ and $r=|x|$.

Setting $B=\left\{x \in \mathbb{R}^{N}:|x|<R(k, N)\right\}$ and using Theorem 2.2, we have that $\int_{B} u w_{k} d x>0$ and that

$$
\begin{aligned}
k^{2} \int_{B} u w_{k} d x & =-\int_{B} u \triangle w_{k} d x=-\int_{\partial B} u \frac{\partial w_{k}}{\partial r} d x-\int_{B} w_{k} \Delta u d x \\
& >\int_{B} w_{k}\{\lambda+f(|x|, u(x))\} u(x) d x \geq \lambda \int_{B} w_{k} u d x
\end{aligned}
$$


Hence $\lambda \leq k^{2}$ for any $k>0$ and so $\lambda \leq 0$.

We turn now to proving that $\lambda>\Lambda$.

Since $g(\infty)>0$ by $(H 2)$, there exists $R>0$ such that

$$
g(r) \geq \frac{1}{2} g(\infty) \text { for all } r \geq R .
$$

By $(H 1)$, there is a $\delta>0$ such that

$$
f(r, s) \leq \frac{1}{4} g(\infty) \text { for all } r \geq 0 \text { and } s \in[0, \delta] .
$$

Also it follows from Theorem 2.2 that there exists $R_{1} \geq R$ such that

$$
0<u(x) \leq \delta \text { for all }|x| \geq R_{1} .
$$

Hence,

$$
f(r, u(x)) \leq \frac{1}{4} g(\infty) \text { for all }|x| \geq R_{1}
$$

and so,

$$
\int_{\mathbb{R}^{N}} f(|x|, u(x)) u(x)^{2} d x<\int_{\mathbb{R}^{N}} g(|x|) u(x)^{2} d x
$$

since $u(x)>0$ for all $x \in \mathbb{R}^{N}$.

Recalling that $u$ satisfies (1.4) for all $v \in H^{1}\left(\mathbb{R}^{N}\right)$, we find that

$$
\begin{aligned}
\int_{\mathbb{R}^{N}}|\nabla u|^{2} d x & =\int_{\mathbb{R}^{N}}\{\lambda+f(|x|, u(x))\} u(x)^{2} d x \\
& <\int_{\mathbb{R}^{N}}\{\lambda+g(|x|)\} u(x)^{2} d x .
\end{aligned}
$$

But, by the definition of $\Lambda$,

$$
\Lambda \int_{\mathbb{R}^{N}} u(x)^{2} d x \leq \int_{\mathbb{R}^{N}}|\nabla u(x)|^{2}-g(|x|) u(x)^{2} d x .
$$

Hence,

$$
\Lambda \int_{\mathbb{R}^{N}} u(x)^{2} d x<\lambda \int_{\mathbb{R}^{N}} u(x)^{2} d x
$$

and we have shown that $\Lambda<\lambda$.\#

Under the hypotheses $(H 1)$ and $(H 2)$, there may or may not be a solution of $(1.1)$ at $\lambda=0$. The next result establishes a context in which there is no solution at $\lambda=0$. The discussion is then completed by constructing a class of functions $f$ for which there is a solution (even a radial solution) at $\lambda=0$. 
Theorem 2.4 Let $p$ be a function satisfying the condition $(B)$ and such that

$$
2 N \int_{0}^{s} p(t) t d t>(N-2) p(s) s^{2} \quad \text { for all } \quad s>0 .
$$

Then the function $f$ defined by

$$
f(r, s)=\left\{\begin{array}{lll}
p(s) & \text { for } & s>0 \\
0 & \text { for } & s=0
\end{array}\right.
$$

satisfies the conditions $(H 1)$ and $(H 2)$ and the problem (1.1) has no solution with $\lambda=0$.

Proof: By Remark 1.5 we already know that $f$ satisfies $(H 1)$ and $(H 2)$. Let $(\lambda, u)$ be a solution of (1.1), then

$$
\int_{\mathbb{R}^{N}}|\nabla u(x)|^{2} d x=\int_{\mathbb{R}^{N}}\{\lambda+p(u(x))\} u(x)^{2} d x,
$$

since (1.4) holds for all $v \in H^{1}\left(\mathbb{R}^{N}\right)$.

By the Pohozaev indentity [10], we also have that

$$
\frac{(N-2)}{2} \int_{\mathbb{R}^{N}}|\nabla u(x)|^{2} d x=N \int_{\mathbb{R}^{N}} \frac{\lambda}{2} u(x)^{2}+P(u(x)) d x,
$$

where $P(s)=\int_{0}^{s} p(t) t d t$.

Combining these two equations we obtain

$$
\frac{(N-2)}{2} \int_{\mathbb{R}^{N}}\left\{\lambda u(x)^{2}+p(u(x)) u(x)^{2}\right\} d x=N \int_{\mathbb{R}^{N}} \frac{\lambda}{2} u(x)^{2}+P(u(x)) d x,
$$

which can be written as

$$
\begin{aligned}
-\lambda \int_{\mathbb{R}^{N}} u(x)^{2} d x & =\int_{\mathbb{R}^{N}} N P(u(x))-\frac{(N-2)}{2} p(u(x)) u(x)^{2} d x \\
& >0
\end{aligned}
$$

since $u(x)>0$ for all $x \in \mathbb{R}^{N}$ by Theorem 2.2. Hence $\lambda<0$ as required. \#

Remark 2.2 Suppose that $p$ satisfies the condition $(B)$ and that there is a number $\gamma \in\left(0, \frac{4}{N-2}\right)$ such that $p^{\prime}(s) s \leq \gamma p(s)$ for all $s>0$. Then, $p$ satisfies the hypotheses of Theorem 2.4 .

In fact, $p^{\prime}(s) s^{2} \leq \gamma p(s) s$ for all $s>0$ and so

$$
\gamma \int_{0}^{s} p(t) t d t \geq \int_{0}^{s} p^{\prime}(t) t^{2} d t=p(s) s^{2}-2 \int_{0}^{s} p(t) t d t
$$


for all $s>0$. Hence

$$
\int_{0}^{s} p(t) t d t \geq \frac{1}{2+\gamma} p(s) s^{2} \quad \text { for } \quad s>0
$$

where $\frac{1}{2+\gamma}>\frac{N-2}{2 N}$.\#

Example 2.1 The function $p(s)=\frac{s^{\sigma}}{1+s^{\sigma}}$ satisfies the hypotheses of Theorem 2.4 provied that $0<\sigma<\frac{4}{N-2}$. Clearly $p$ satisfies $(B)$ for all $\sigma>0$. Since $p^{\prime}(s) s=\frac{\sigma s^{\sigma}}{\left(1+s^{\sigma}\right)^{2}} \leq \sigma p(s)$ for all $s>0$, the preceding remark establishes the claim.

Theorem 2.5 Let $p$ be a function satisfying the condition $(B)$ such that $p(s)=s^{\sigma}$ for $0<s \leq 1$, where $\frac{2}{N-2}<\sigma<\frac{4}{N}$. Set

$$
q(r)=A+\frac{B}{1+r^{2}} \quad \text { for } \quad r \geq 0
$$

where $A=\frac{2}{\sigma}\left\{N-2-\frac{2}{\sigma}\right\}$ and $B=\frac{4(1+\sigma)}{\sigma^{2}}$. Let

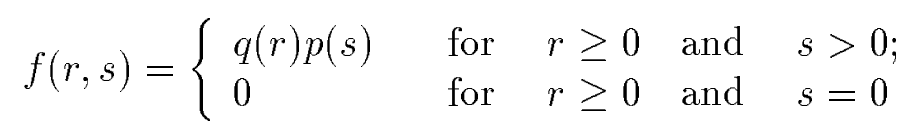

and

$$
u(x)=\left(1+|x|^{2}\right)^{-\frac{1}{\sigma}} \quad \text { for } \quad x \in \mathbb{R}^{N} .
$$

Then $f$ satisfies the conditions $(H 1)$ and $(H 2)$ and $(0, u)$ is a solution of (1.1).

Proof: The restrictions on $\sigma$ ensure that $A>0$ and $B>0$. Hence the function $q$ satisfies the condition $(A)$ and consequently the function $f$ satisfies $(H 1)$ and $(H 2)$ by Remark 1.5 .

Setting $r=|x|$, straight forward calculations show that

$$
\begin{aligned}
\triangle u(x) & =\left\{\frac{4}{\sigma}\left(\frac{1}{\sigma}+1\right) r^{2}\left(1+r^{2}\right)^{-1}-\frac{2 N}{\sigma}\right\}\left(1+r^{2}\right)^{-\left(\frac{1}{\sigma}+1\right)} \\
& =-q(r) u(x)^{\sigma+1}=-f(r, u(x)) u(x)
\end{aligned}
$$

since $0<u(x) \leq 1$ for all $x \in \mathbb{R}^{N}$.

Furthermore $u \in H^{1}\left(\mathbb{R}^{N}\right)$ since $\sigma<\frac{4}{N}$. Thus, $(0, u)$ is a solution of (1.1).\# 
Remark 2.3 Functions satisfying the hypotheses of Theorem 2.5 exist if and only if $N \geq 5$.\#

\section{Existence of radially symmetric solutions}

In this section we show that for every $\lambda \in(\Lambda, 0)$ there is a radially symmetric solution of (1.1). These solutions are obtained from critical points of a functional $J$ defined on the Hilbert space, $(H,\|\cdot\|)$, where

$$
H=H_{0}^{1}(0, \infty)=\left\{u \in L^{2}(0, \infty): u^{\prime} \in L^{2}(0, \infty) \text { and } u(0)=0\right\}
$$

and

$$
\|u\|=\left\{|u|_{2}^{2}+\left|u^{\prime}\right|_{2}^{2}\right\}^{\frac{1}{2}} .
$$

We recall that for any $u \in H$,

$$
\begin{gathered}
u \in C([0, \infty)) \cap L^{\infty}(0, \infty) \text { with } \quad|u|_{\infty}^{2} \leq 2|u|_{2}\left|u^{\prime}\right|_{2} \leq\|u\|^{2}, \\
|u(r)| \leq r^{\frac{1}{2}}\left|u^{\prime}(r)\right|_{2}, \quad \text { for all } r \geq 0 \\
\lim _{r \rightarrow 0} r^{-\frac{1}{2}} u(r)=\lim _{r \rightarrow \infty} u(r)=0 \\
\int_{0}^{\infty} \frac{u(r)^{2}}{r^{2}} d r \leq 4\left|u^{\prime}(r)\right|_{2}^{2} \text { (Hardy's inequality) }
\end{gathered}
$$

and

$$
\int_{0}^{\infty} \frac{u(r) u^{\prime}(r)}{r} d r=\lim _{\substack{a \rightarrow 0 \\ b \rightarrow \infty}} \int_{a}^{b} \frac{\left\{u(r)^{2}\right\}^{\prime}}{2 r} d r=\frac{1}{2} \int_{0}^{\infty} \frac{u(r)^{2}}{r^{2}} d r
$$

For $\lambda<0$, we set

$$
\|u\|_{\lambda}=\left\{\int_{0}^{\infty} u^{\prime}(r)^{2}+|\lambda| u(r)^{2}+\frac{(N-1)(N-3)}{4} \frac{u(r)^{2}}{r^{2}} d r\right\}^{\frac{1}{2}}
$$

and observe that

$$
\min \{1,|\lambda|\}\|u\|^{2} \leq\|u\|_{\lambda} \leq \max \left\{(N-2)^{2},|\lambda|\right\}\|u\|^{2},
$$

for all $u \in H$, so that $\|\cdot\|_{\lambda}$ is an equivalent norm to $\|\cdot\|$ on $H$. 
For a function $f$ which satisfies the conditions $(H 1)$ and $(H 2)$, we set

$$
F_{1}(r, s)=\int_{0}^{s} f\left(r, r^{\frac{1-N}{2}} t^{+}\right) t d t=r^{N-1} F\left(r, r^{\frac{1-N}{2}} s^{+}\right),
$$

for $r>0$ and $s \in \mathbb{R}$, where $t^{+}=\max \{0, t\}$ and $F(r, s)=\int_{0}^{s} f(r, t) t d t$ for $r, s \geq 0$ as in Sections 1 and 2 .

Clearly $0 \leq F_{1}(r, s) \leq \frac{1}{2}|g|_{\infty}\left(s^{+}\right)^{2}$ for all $r>0$ and $s \in \mathbb{R}$. Hence, for any $\lambda<0$, we can define a functional $J: H \longrightarrow \mathbb{R}$ by

$$
J(u)=\frac{1}{2}\|u\|_{\lambda}^{2}-\int_{0}^{\infty} F_{1}(r, u(r)) d r \quad \text { for } \quad u \in H .
$$

Furthermore, it is easy to show that $J \in C^{1}(H, \mathbb{R})$ with

$$
\begin{aligned}
J^{\prime}(u) v= & \int_{0}^{\infty}\left\{u^{\prime}(r) v^{\prime}(r)-\lambda u(r) v(r)+\frac{(N-1)(N-3)}{4 r^{2}} u(r) v(r)\right\} d r \\
& -\int_{0}^{\infty} f\left(r, r^{\frac{1-N}{2}} u(r)^{+}\right) u(r) v(r) d r
\end{aligned}
$$

for all $u, v \in H$.

Lemma 3.1 Consider $\lambda<0$ and a function $f$ satisfying the conditions $(H 1)$ and (H2). Suppose that $u \in H \backslash\{0\}$ and $J^{\prime}(u)=0$. Then, $u \in C^{2}((0, \infty))$ with $u(r) \geq 0$ and

$$
u^{\prime \prime}(r)=\left\{\frac{(N-1)(N-3)}{4 r^{2}}-\lambda-f\left(r, r^{\frac{1-N}{2}} u(r)\right)\right\} u(r)
$$

for all $r>0$. Furthermore,

$$
r^{\frac{N}{2}-2}\left\{r^{\frac{1-N}{2}} u(r)\right\}^{\prime} \longrightarrow 0 \quad \text { as } \quad r \rightarrow 0 .
$$

Proof: Since $J^{\prime}(u) v=0$ for all $v \in C_{0}^{\infty}((0, \infty)) \subset H$, we see that $u^{\prime}$ has a weak derivative on $(0, \infty)$ and $u^{\prime \prime}(r)=h(r)$ a.e. on $(0, \infty)$ where

$$
h(r)=\left\{\frac{(N-1)(N-3)}{4 r^{2}}-\lambda-f\left(r, r^{\frac{1-N}{2}} u(r)^{+}\right)\right\} u(r) .
$$

From $(H 1)$ and the continuity of $u$ on $[0, \infty)$ it follows that $h \in C((0, \infty))$ and consequently $u \in C^{2}((0, \infty))$. If $u\left(r_{0}\right)<0$ for some $r_{0}>0$, we have that

$$
u^{\prime \prime}\left(r_{0}\right)=h\left(r_{0}\right)=\left\{\frac{(N-1)(N-3)}{4 r_{0}^{2}}-\lambda\right\} u\left(r_{0}\right)<0
$$

and so $u$ cannot have a negative minimum in $(0, \infty)$. Since $\lim _{r \rightarrow 0} u(r)=\lim _{r \rightarrow \infty} u(r)=$ 0 this implies that $u(r) \geq 0$ for all $r>0$. Thus $u(r)^{+}=u(r)$ for all $r>0$.

Using the variation of constants formula for the equation

$$
-u^{\prime \prime}(r)+\frac{(N-1)(N-3)}{4 r^{2}} u(r)=k(r) \quad \text { on } \quad(0, \infty)
$$


where $k(r)=\left\{\lambda+f\left(r, r^{\frac{1-N}{2}} u(r)\right)\right\} u(r)$, we find that there are constants $A$ and $B$ such that

$u(r)=r^{\frac{N-1}{2}}\left\{A-\int_{1}^{r} \frac{s^{\frac{3-N}{2}} k(s)}{N-2} d s\right\}+r^{\frac{3-N}{2}}\left\{B+\int_{1}^{r} \frac{s^{\frac{N-1}{2}} k(s)}{N-2} d s\right\} \quad$ for $\quad r>0$.

Note that $k \in C((0, \infty))$ and $|k(r)| \leq\left\{|\lambda|+|g|_{\infty}\right\} u(r) \leq \psi(r) r^{\frac{1}{2}}$, where $\psi(r)=\left\{|\lambda|+|g|_{\infty}\right\}\left\{\int_{0}^{r} u^{\prime}(s)^{2} d s\right\}^{\frac{1}{2}} \longrightarrow 0$ as $r \rightarrow 0$. It follows that

$$
\lim _{r \rightarrow 0} r^{\frac{N-1}{2}} \int_{1}^{r} s^{\frac{3-N}{2}} k(s) d s=0,
$$

and since $\lim _{r \rightarrow 0} u(r)=0$ this means that

$$
\lim _{r \rightarrow 0}\left\{B+\int_{1}^{r} \frac{s^{\frac{N-1}{2}} k(s)}{N-2} d s\right\}=0
$$

Hence $B=\int_{0}^{1} \frac{s^{\frac{N-1}{2}} k(s)}{N-2} d s$ and

$$
u(r)=r^{\frac{N-1}{2}}\left\{A-\int_{1}^{r} \frac{s^{\frac{3-N}{2}} k(s)}{N-2} d s\right\}+r^{\frac{3-N}{2}} \int_{0}^{r} \frac{s^{\frac{N-1}{2}} k(s)}{N-2} d s .
$$

Thus

$$
\left\{r^{\frac{1-N}{2}} u(r)\right\}^{\prime}=-r^{1-N} \int_{0}^{r} s^{\frac{N-1}{2}} k(s) d s
$$

and

$$
\left|r^{\frac{N}{2}-2}\left\{r^{\frac{N-1}{2}} u(r)\right\}^{\prime}\right| \leq r^{-\left(\frac{N}{2}+1\right)} \int_{0}^{r} s^{\frac{N-1}{2}} \psi(s) s^{\frac{1}{2}} d s \leq \frac{2 \Psi(r)}{N+2}
$$

where $\Psi(r)=\max _{0<s \leq r} \psi(s) \longrightarrow 0$ as $r \rightarrow 0$.\#

Theorem 3.2 Under the hypotheses of Lemma 3.1 we set

$$
w(x)=r^{\frac{1-N}{2}} u(r) \quad \text { for } \quad x \in \mathbb{R}^{N} \text { with } \quad r=|x|>0 .
$$

Then $(\lambda, w)$ is a solution of (1.1).

Proof: By Lemma 3.1, $w \in C^{2}\left(\mathbb{R}^{N} \backslash\{0\}\right)$ and

$$
\begin{gathered}
\partial_{i} w(x)=\frac{x_{i}}{r}\left\{\frac{1-N}{2 r} u(r)+u^{\prime}(r)\right\} r^{\frac{1-N}{2}} \quad \text { for } \quad x \neq 0, \\
|\nabla w(x)|^{2}=r^{1-N}\left\{\frac{(N-1)^{2}}{4} \frac{u(r)^{2}}{r^{2}}+u^{\prime}(r)^{2}-(N-1) \frac{u(r) u^{\prime}(r)}{r}\right\}
\end{gathered}
$$


and

$$
\begin{aligned}
& \int_{\mathbb{R}^{N}} w(x)^{2}+|\nabla w(x)|^{2} d x=\omega_{N} \int_{0}^{\infty} r^{N-1}\left\{w(x)^{2}+|\nabla w(x)|^{2}\right\} d r \\
= & \omega_{N} \int_{0}^{\infty} u(r)^{2}+\frac{(N-1)^{2}}{4} \frac{u(r)^{2}}{r^{2}}+u^{\prime}(r)^{2}-(N-1) \frac{u(r) u^{\prime}(r)}{r} d r \\
= & \omega_{N} \int_{0}^{\infty} u(r)^{2}+\frac{(N-1)(N-3)}{4} \frac{u(r)^{2}}{r^{2}}+u^{\prime}(r)^{2} d r, \text { by }(3.5) \\
\leq & (N-2)^{2} \omega_{N}\|u\|^{2}, \text { by }(3.7),
\end{aligned}
$$

where $\omega_{N}$ is the $(N-1)$-dimensional surface measure of $\left\{x \in \mathbb{R}^{N}:|x|=1\right\}$. In particular, $w$ and $\partial_{i} w \in L^{2}\left(\mathbb{R}^{N}\right)$ for $i=1, \cdots, N$, and, for any $\varphi \in C_{0}^{\infty}\left(\mathbb{R}^{N}\right)$,

$$
\begin{aligned}
& \int_{\mathbb{R}^{N}} w(x) \partial_{i} \varphi(x) d x=\lim _{\varepsilon \rightarrow 0} \int_{|x| \geq \varepsilon} w(x) \partial_{i} \varphi(x) d x \\
= & \lim _{\varepsilon \rightarrow 0}\left\{-\int_{|x|=\varepsilon} \varepsilon^{\frac{1-N}{2}} u(\varepsilon) \varphi(x) \frac{x_{i}}{\varepsilon} d x-\int_{|x| \geq \varepsilon} \partial_{i} w(x) \varphi(x) d x\right\}
\end{aligned}
$$

Now

$$
\begin{aligned}
& \left|\int_{|x|=\varepsilon} \varepsilon^{\frac{1-N}{2}} u(\varepsilon) \varphi(x) \frac{x_{i}}{\varepsilon} d x\right| \leq \varepsilon^{\frac{1-N}{2}} u(\varepsilon) \omega_{N} \varepsilon^{N-1} \max _{|x|=\varepsilon}|\varphi(x)| \\
= & \omega_{N} \varepsilon^{\frac{N-1}{2}} u(\varepsilon) \max _{|x|=\varepsilon}|\varphi(x)|
\end{aligned}
$$

so

$$
\lim _{\varepsilon \rightarrow 0} \int_{|x|=\varepsilon} \varepsilon^{\frac{1-N}{2}} u(\varepsilon) \varphi(x) \frac{x_{i}}{\varepsilon} d x=0 .
$$

This shows that $\partial_{i} w$ is the weak derivetive of $w$ on $\mathbb{R}^{N}$. It follows that $w \in$ $H^{1}\left(R^{N}\right)$ and that for $v \in C_{0}^{\infty}\left(\mathbb{R}^{N}\right)$

$$
\int_{\mathbb{R}^{N}} \nabla w \cdot \nabla v d x=\lim _{\varepsilon \rightarrow 0}\left\{-\int_{|x|=\varepsilon} \frac{\partial w}{\partial r} v d x-\int_{|x| \geq \varepsilon} \Delta w v d x\right\} .
$$

But

$$
r^{N-1} \frac{\partial w(x)}{\partial r}=r^{N-1}\left(r^{\frac{1-N}{2}} u(r)\right)^{\prime} \longrightarrow 0 \quad \text { as } \quad r \rightarrow 0
$$

by Lemma 3.1 since $N-1 \geq \frac{N}{2}-2$ and so

$$
\lim _{\varepsilon \rightarrow 0}\left\{-\int_{|x|=\varepsilon} \frac{\partial w}{\partial r} v d x\right\}=0 .
$$

Furthermore, for $r>0$,

$$
\begin{aligned}
\triangle w(r) & =w^{\prime \prime}(r)+\frac{N-1}{r} w^{\prime}(r) \\
& =r^{\frac{1-N}{2}}\left\{u^{\prime \prime}(r)-\frac{(N-1)(N-3)}{4 r^{2}} u(r)\right\} \\
& =-r^{\frac{1-N}{2}}\left\{\lambda+f\left(r, r^{\frac{1-N}{2}} u(r)\right)\right\} u(r) \\
& =-\{\lambda+f(|x|, w(x))\} w(x)
\end{aligned}
$$


and

$$
\lim _{\varepsilon \rightarrow 0} \int_{|x| \geq \varepsilon} \triangle w v d x=-\int_{\mathbb{R}^{N}}\{\lambda+f(|x|, w(x))\} w(x) v(x) d x
$$

Thus

$$
\int_{\mathbb{R}^{N}} \nabla w \cdot \nabla v d x=\int_{\mathbb{R}^{N}}\{\lambda+f(|x|, w(x))\} w(x) v(x) d x
$$

for all $v \in C_{0}^{\infty}\left(\mathbb{R}^{N}\right)$ as required.\#

Corollary 3.3 Under the hypotheses of Lemma 3.1 we have that $u(r)>0$ for all $r>0$. Furthermore there is an $\ell \in(0, \infty)$ such that

$$
\lim _{r \rightarrow 0} r^{\frac{1-N}{2}} u(r)=\frac{2}{N-1} \lim _{r \rightarrow 0} r^{\frac{3-N}{2}} u^{\prime}(r)=\ell .
$$

Proof: From Theorems 2.2 and 3.2, we have that $w \in C^{1}\left(\mathbb{R}^{N}\right)$ and $w(x)>$ 0 for all $x \in \mathbb{R}^{N}$, where $w(x)=r^{\frac{1-N}{2}} u(r)$ and

$$
\frac{\partial w(x)}{\partial r}=\frac{1-N}{2} r^{\frac{1-N}{2}-1} u(r)+r^{\frac{1-N}{2}} u^{\prime}(r)
$$

for $|x|=r>0$. Setting $\ell=w(0)$, the conclusions follow immediately.\#

To establish the existence of a non-zero critical point of $J$ we use a variant of the mountain pass theorem, [1], which we now recall.\#

Propositon 3.4 Let $E$ be a real Hilbert space and consider a functional $J \in(E, \mathbb{R})$ satisfying the following conditions.

(a) There exist $\rho>0$ and $\alpha>0$ such that $J(u) \geq \alpha$ for all $u \in E$ with $\|u\|=\rho$.

(b) $J(0)=0$ and there exists $e \in E$ with $\|e\|>\rho$ such that $J(e) \leq 0$.

(c) For any $c>0$, every bounded sequence $\left\{u_{n}\right\} \subset E$ such that $J\left(u_{n}\right) \longrightarrow c$ and $J^{\prime}\left(u_{n}\right) \longrightarrow 0$ in $E^{*}$ as $n \rightarrow \infty$ possesses a convergent subsequence.

(d) For any $c>0$, there exist constants $\delta, R, \eta>0$ such that

$$
\left\|J^{\prime}(u)\right\|\|u\| \geq \eta \text { for all } u \text { such that } J(u) \in[c-\delta, c+\delta] \text { and }\|u\| \geq R .
$$

Then there is an element $u \in E$ such that $J(u) \geq \alpha$ and $J^{\prime}(u)=0$.\# 
By Theorem 3.2, we obtain solutions of problem (1.1) provided that Proposition 3.4 can be applied to the functional defined by (3.9). As the next result shows, it is in establishing the properties (a) and (b) that the restrictions $\lambda<0$ and $\lambda>\Lambda$ appear naturally.

Lemma 3.5 Consider a function $f$ satisfying the conditions $(H 1)$ and $(H 2)$.

(a) For any $\lambda<0$, there exist $\rho(\lambda)>0$ and $\alpha(\lambda)>0$ such that $J(u) \geq \alpha(\lambda)$ for all $u \in H$ with $\|u\|_{\lambda}=\rho(\lambda)$.

(b) For any $\lambda \in(\Lambda, 0)$, there exists $e_{\lambda} \in H$ with such that $\left\|e_{\lambda}\right\|_{\lambda}>\rho(\lambda)$ and $J\left(e_{\lambda}\right) \leq 0$.

Proof: (a) Let $\sigma=\frac{4}{N-1}$. Choose $\varepsilon=\frac{1}{2} \min \{1,|\lambda|\}$. By $(H 1)$, there exists $S_{\varepsilon}>0$ such that

$$
0 \leq f(r, s) \leq \varepsilon \forall r \geq 0 \quad \text { and } \quad 0 \leq s \leq S_{\varepsilon}
$$

By $(H 2)$, there is a constant $C_{\varepsilon}>0$ such that

$$
C_{\varepsilon} s^{\sigma} \geq C_{\varepsilon} S_{\varepsilon}^{\sigma} \geq|g|_{\infty} \geq f(r, s) \geq 0 \text { for all } r \geq 0 \text { and } s \geq S_{\varepsilon} .
$$

Hence

$$
0 \leq f(r, s) \leq \varepsilon+C_{\varepsilon} s^{\sigma} \text { for all } \quad r \geq 0 \quad \text { and } \quad s \geq 0 .
$$

Using (3.8), this means that

$$
\begin{aligned}
0 \leq F_{1}(r, s) & =r^{N-1} F\left(r, r^{\frac{1-N}{2}} s^{+}\right) \\
& \leq r^{N-1}\left\{\frac{\varepsilon}{2}\left(r^{\frac{1-N}{2}} s^{+}\right)^{2}+\frac{C_{\varepsilon}}{\sigma+2}\left(r^{\frac{1-N}{2}} s^{+}\right)^{\sigma+2}\right\} \\
& \leq \frac{\varepsilon}{2} s^{2}+\frac{C_{\varepsilon}}{\sigma+2}\left(\frac{s}{r}\right)^{2}\left|s^{+}\right|^{\sigma}
\end{aligned}
$$

for all $r \geq 0$ and $s \in \mathbb{R}$.

Hence, for $u \in H$,

$$
\begin{aligned}
\int_{0}^{\infty} F_{1}(r, u(r)) d r & \leq \frac{\varepsilon}{2}|u|_{2}^{2}+\frac{C_{\varepsilon}}{\sigma+2}|u|_{\infty}^{\sigma} 4\left|u^{\prime}\right|_{2}^{2} \\
& \leq\left\{\frac{\varepsilon}{2}+\frac{C_{\varepsilon}}{\sigma+2}\|u\|^{\sigma}\right\}\|u\|^{2} \text { by }
\end{aligned}
$$

and so, by (3.7)

$$
J(u) \geq \frac{1}{2} \min \{1,|\lambda|\}\|u\|^{2}-\left\{\frac{\varepsilon}{2}+\frac{C_{\varepsilon}}{\sigma+2}\|u\|^{\sigma}\right\}\|u\|^{2}
$$




$$
\begin{aligned}
& =\left\{\frac{\varepsilon}{2}-\frac{C_{\varepsilon}}{\sigma+2}\|u\|^{\sigma}\right\}\|u\|^{2} \\
& \geq \frac{\varepsilon}{4}\|u\|^{2} \text { if } \quad\|u\|^{\sigma} \leq \frac{(\sigma+2) \varepsilon}{4 C_{\varepsilon}} .
\end{aligned}
$$

This proves that $J$ has the property (a).

(b) As noted in the introduction $\Lambda \leq-g(\infty)<0$ and our way of finding an approriate element $e \in H$ depends on which of the following cases occurs:

$$
\operatorname{CASE}(\mathrm{I}): \Lambda<-g(\infty) \text { or } \operatorname{CASE}(\mathrm{II}): \Lambda=-g(\infty)
$$

CASE(I): In this case, $\Lambda$ is a simple eigenvalue of the operator $S$ and there exists a positive eigenfunction $\varphi \in H^{2}$ such that $S \varphi=\Lambda \varphi$ on $\mathbb{R}^{N}$ and $\lim _{|x| \rightarrow \infty} e^{\gamma|x|} \varphi(x)=0$ for any $\gamma<\sqrt{|\Lambda|}$ (see, [2]). Furthermore, by applying the same agruments as in Theorem 2.2 , we see that $\varphi \in C^{1}\left(\mathbb{R}^{N}\right)$ with $\varphi(x)>0$ for all $x \in \mathbb{R}^{N}$. Finally, denoting the spherical average of $\varphi$ by $\tilde{\varphi}$ and using the fact that $\triangle \tilde{\varphi}=(\widetilde{\triangle \varphi})$ (see $[3])$ and $g \tilde{\varphi}=\widetilde{(g \varphi})$, it follows that $\varphi=\tilde{\varphi}$. Hence there is a function $w:[0, \infty) \longrightarrow(0, \infty)$ such that $\varphi(x)=w(|x|)$ for all $x \in \mathbb{R}^{N}$ and $\lim _{r \rightarrow \infty} e^{\gamma r} w(r)=0$ for all $\gamma<\sqrt{|\Lambda|}$.

Setting $\psi(r)=r^{\frac{N-1}{2}} w(r)$ for $r>0$, we claim that

$$
\psi \in H \quad \text { and } \quad\|\psi\|_{\lambda}^{2}=\frac{1}{\omega_{N}} \int_{\mathbb{R}^{N}}|\nabla \varphi(x)|^{2}+|\lambda| \varphi(x)^{2} d x
$$

In fact,

$$
\int_{\mathbb{R}^{N}} \varphi(x)^{2} d x=\omega_{N} \int_{0}^{\infty} r^{N-1} w(r)^{2} d r=\omega_{N} \int_{0}^{\infty} \psi(r)^{2} d r
$$

and

$$
\begin{aligned}
\int_{\mathbb{R}^{N}}|\nabla \varphi(x)|^{2} d x & =\omega_{N} \int_{0}^{\infty} r^{N-1} w^{\prime}(r)^{2} d r \\
& =\omega_{N} \int_{0}^{\infty}\left\{\psi^{\prime}(r)-\frac{N-1}{2} \frac{\psi}{r}\right\}^{2} d r \\
& =\omega_{N} \int_{0}^{\infty} \psi^{\prime}(r)^{2}-\frac{N-1}{2 r}\left\{\psi(r)^{2}\right\}^{\prime}+\frac{(N-1)^{2}}{4} \frac{\psi^{2}}{r^{2}} d r \\
& =\omega_{N} \int_{0}^{\infty} \psi^{\prime}(r)^{2}+\frac{(N-1)(N-3)}{4 r^{2}} \psi(r)^{2} d r,
\end{aligned}
$$

since $\lim _{r \rightarrow 0} \frac{\psi^{2}}{r}=\lim _{r \rightarrow \infty} \frac{\psi^{2}}{r}=0$.

In particular, $\|\psi\|_{\lambda}<\infty$ and $\lim _{r \rightarrow 0} \psi(r)=0$ so $\psi \in H$. Furthermore, since 
$\varphi \in H^{2}\left(R^{N}\right)$, we have that

$$
\begin{aligned}
\|\psi\|_{\lambda}^{2} & =\frac{1}{\omega_{N}} \int_{\mathbb{R}^{N}}\{(-\Delta \varphi)+|\lambda| \varphi\} \varphi d x \\
& =\frac{1}{\omega_{N}} \int_{\mathbb{R}^{N}}\{\Lambda-\lambda+g(|x|)\} \varphi(x)^{2} d x \\
& =\int_{0}^{\infty}\{\Lambda-\lambda+g(r)\} \psi(r)^{2} d r .
\end{aligned}
$$

We now show that there is a number $t>0$ such that $J(t \psi)<0$. For any $t>0$ we have that

$$
t^{-2} J(t \psi)=\frac{1}{2}\|\psi\|_{\lambda}^{2}-t^{-2} \int_{0}^{\infty} F_{1}(r, t \psi(r)) d r
$$

where

$$
\begin{aligned}
F_{1}(r, t \psi(r)) & =\int_{0}^{t \psi(r)} f\left(r, r^{\frac{1-N}{2}} s\right) s d s \\
& =\frac{t^{2} \psi(r)^{2}}{2} \int_{0}^{1} f\left(r, r^{\frac{1-N}{2}} \psi(r) t \sqrt{\tau}\right) d \tau \quad \text { with } \quad \sqrt{\tau}=\frac{s}{t \psi(r)}
\end{aligned}
$$

and

$$
\begin{aligned}
t^{-2} \int_{0}^{\infty} F_{1}(r, t \psi(r)) d r & =\frac{1}{2} \int_{0}^{\infty} \varphi(r)^{2} \int_{0}^{1} f\left(r, r^{\frac{1-N}{2}} \psi(r) t \sqrt{\tau}\right) d \tau d r \\
& \longrightarrow \frac{1}{2} \int_{0}^{\infty} g(r) \psi(r)^{2} d r \quad \text { as } \quad t \rightarrow \infty
\end{aligned}
$$

by dominated convergence.

Hence, by (3.11) to (3.13),

$$
\begin{aligned}
\lim _{t \rightarrow \infty} t^{-2} J(t \psi) & =\frac{1}{2}\left\{\|\psi\|_{\lambda}^{2}-\frac{1}{2} \int_{0}^{\infty} g(r) \psi(r)^{2} d r\right\} \\
& =\frac{1}{2}(\Lambda-\lambda) \int_{0}^{\infty} \psi(r)^{2} d r<0 \quad \text { for } \quad \lambda>\Lambda
\end{aligned}
$$

This proves part (b) when CASE (I) occurs.

CASE(II): In this case $\Lambda$ may not be an eigenvalue of $S$ so that the eigenfunction used for the construction of $e$ in CASE (I) is not available.

For any $\alpha>0$, let $v_{\alpha}(r)=2 \alpha^{\frac{3}{2}} r e^{-\alpha r}$ for $r>0$. It is easy to check that for all $\alpha>0$,

$$
v_{\alpha} \in H \quad \text { with } \quad\left|v_{\alpha}\right|_{2}=1,\left|v_{\alpha}^{\prime}\right|_{2}=\alpha
$$


and

$$
\left\|v_{\alpha}\right\|_{\lambda}^{2}=\alpha^{2}+|\lambda|+\frac{(N-1)(N-3)}{2} \alpha^{2}=\frac{(N-2)^{2}+1}{2} \alpha^{2}+|\lambda| .
$$

Thus for any $\alpha, t>0$ we have that

$$
t^{-2} J\left(t v_{\alpha}\right)=\frac{1}{2}\left\|v_{\alpha}\right\|_{\lambda}^{2}-t^{-2} \int_{0}^{\infty} F_{1}\left(r, t v_{\alpha}(r)\right) d r,
$$

where

$$
\begin{aligned}
F_{1}\left(r, t v_{\alpha}(r)\right) & =\int_{0}^{t v_{\alpha}(r)} f\left(r, r^{\frac{1-N}{2}} s\right) s d s \\
& =\frac{t^{2} v_{\alpha}(r)^{2}}{2} \int_{0}^{1} f\left(r, r^{\frac{1-N}{2}} v_{\alpha}(r) t \sqrt{\tau}\right) d \tau \quad \text { with } \quad \sqrt{\tau}=\frac{s}{t v_{\alpha}(r)}
\end{aligned}
$$

and

$$
\begin{aligned}
t^{-2} \int_{0}^{\infty} F_{1}\left(r, t v_{\alpha}(r)\right) d r & =\frac{1}{2} \int_{0}^{\infty} v_{\alpha}(r)^{2} \int_{0}^{1} f\left(r, r^{\frac{1-N}{2}} v_{\alpha}(r) t \sqrt{\tau}\right) d \tau d r \\
& \longrightarrow \frac{1}{2} \int_{0}^{\infty} g(r) v_{\alpha}(r)^{2} d r \text { as } \quad t \rightarrow \infty
\end{aligned}
$$

by dominated convergence.

Thus,

$\lim _{t \rightarrow \infty} t^{-2} J\left(t v_{\alpha}\right)=\frac{1}{2}\left\{\frac{(N-2)^{2}+1}{2} \alpha^{2}+|\lambda|\right\}-\frac{1}{2} \int_{0}^{\infty} g(r) v_{\alpha}(r)^{2} d r \quad$ for any $\quad \alpha>0$.

But

$$
\begin{aligned}
\int_{0}^{\infty} g(r) v_{\alpha}(r)^{2} d r & =\int_{0}^{\infty} g(r) 4 \alpha^{3} r^{2} e^{-2 \alpha r} d r \\
& =\int_{0}^{\infty} g\left(\frac{\rho}{\alpha}\right) 4 \rho^{2} e^{-2 \rho} d \rho \\
& \longrightarrow 4 g(\infty) \int_{0}^{\infty} \rho^{2} e^{-2 \rho} d \rho=g(\infty)
\end{aligned}
$$

as $\alpha \rightarrow 0$ by dominated convergence.

For $\lambda \in(-g(\infty), 0],|\lambda|-g(\infty)<0$ and there exists $\alpha(\lambda)>0$ such that

$$
\int_{0}^{\infty} g(r) v_{\alpha}(r)^{2} d r>g(\infty)+\frac{1}{2}\{|\lambda|-g(\infty)\} \quad \text { for all } \quad \alpha \in(0, \alpha(\lambda)] .
$$

Hence for $\alpha \in(0, \alpha(\lambda)]$

$$
\begin{aligned}
\lim _{t \rightarrow \infty} t^{-2} J\left(t v_{\alpha}\right) & \leq \frac{1}{2}\left\{\frac{(N-2)^{2}+1}{2} \alpha^{2}+|\lambda|\right\}-\frac{1}{2} g(\infty)-\frac{1}{4}\{|\lambda|-g(\infty)\} \\
& =\frac{1}{4}\left[(N-2)^{2}+1\right] \alpha^{2}+\frac{1}{4}\{|\lambda|-g(\infty)\}
\end{aligned}
$$


Thus we see that there exists $\alpha_{1}(\lambda) \in(0, \alpha(\lambda)]$ such that

$$
\lim _{t \rightarrow \infty} t^{-2} J\left(t v_{\alpha}\right) \leq \frac{1}{8}\{|\lambda|-g(\infty)\}<0 \quad \text { for } \quad \alpha=\alpha_{1}(\lambda)
$$

and we can set $e=t v_{\alpha_{1}(\lambda)}$ for some sufficiently large value of $t$. This completes the proof of the lemma.\#

Theorem 3.6 Let $f$ be a function satisfying the conditions $(H 1)$ and $(H 2)$. For every $\lambda \in(\Lambda, 0)$ there exists a solution $(\lambda, u)$ of problem (1.1) where $u$ is radially symmetric.

Proof: By Lemma 3.1 and Theorem 3.2, it is enough to show that the functional $J$ satisfies the conditions of Proposition 3.4. In Lemma 3.5 we have already established that $J$ has the properties (a) and (b).

(c): Let $\left\{u_{n}\right\}$ be a bounded sequence in $H$ such that

$$
J^{\prime}\left(u_{n}\right) \longrightarrow 0 \quad \text { as } \quad n \rightarrow \infty .
$$

Suppose that $\left\|u_{n}\right\|_{\lambda} \leq K$ for all $n \in I N$ and some $K>0$. By passing to a subsequence we can suppose that there is an element $u \in H$ such that

$$
u_{n} \stackrel{n}{\rightarrow} u \quad \text { weakly in } H .
$$

We also have that, for all $\varphi \in H$,

$$
J^{\prime}\left(u_{n}\right) \varphi \longrightarrow 0 \quad \text { and } \quad J^{\prime}\left(u_{n}\right) u_{n} \longrightarrow 0 \quad \text { as } \quad n \rightarrow \infty .
$$

Thus

$$
\int_{0}^{\infty} u_{n}^{\prime} u^{\prime}+|\lambda| u_{n} u+\frac{(N-1)(N-3)}{4 r^{2}} u_{n} u-f\left(r, r^{\frac{1-N}{2}} u_{n}(r)^{+}\right) u_{n} u d r=\circ(1)
$$

and

$$
\left\|u_{n}\right\|_{\lambda}^{2}-\int_{0}^{\infty} f\left(r, r^{\frac{1-N}{2}} u_{n}(r)^{+}\right) u_{n}(r)^{2} d r=\circ(1)
$$

where $\circ(1)$ denotes a quantity which tends to zero as $n \rightarrow \infty$.

Since $u_{n} \stackrel{n}{\rightarrow} u$ weakly in $H$ we have that

$$
\int_{0}^{\infty} u_{n}^{\prime} u^{\prime}+|\lambda| u_{n} u+\frac{(N-1)(N-3)}{4 r^{2}} u_{n} u d r \stackrel{n}{\longrightarrow}\|u\|_{\lambda}^{2} .
$$

Hence we have that

$$
\left\|u_{n}\right\|_{\lambda}^{2}-\|u\|_{\lambda}^{2}=\int_{0}^{\infty} f\left(r, r^{\frac{1-N}{2}} u_{n}(r)^{+}\right) u_{n}(r)\left[u_{n}(r)-u(r)\right] d r+\circ(1)
$$


where for any $R>0$

$$
\begin{aligned}
& \left|\int_{0}^{R} f\left(r, r^{\frac{1-N}{2}} u_{n}(r)^{+}\right) u_{n}(r)\left[u_{n}(r)-u(r)\right] d r\right| \\
\leq & |g|_{\infty}\left\{\int_{0}^{R} u_{n}(r)^{2} d r\right\}^{\frac{1}{2}}\left\{\int_{0}^{R}\left[u_{n}(r)-u(r)\right]^{2} d r\right\}^{\frac{1}{2}} \\
\leq & |\lambda|^{-\frac{1}{2}}|g|_{\infty} K\left\{\int_{0}^{R}\left[u_{n}(r)-u(r)\right]^{2} d r\right\}^{\frac{1}{2}}
\end{aligned}
$$

and, since $\left|u_{n}\right|_{\infty} \leq\|u\| \leq(\min \{1,|\lambda|\})^{-\frac{1}{2}}\left\|u_{n}\right\|_{\lambda}$,

$$
\begin{aligned}
& \left|\int_{R}^{\infty} f\left(r, r^{\frac{1-N}{2}} u_{n}(r)^{+}\right) u_{n}(r)\left[u_{n}(r)-u(r)\right] d r\right| \\
\leq & \sup _{r \geq R} f\left(r, R^{\frac{1-N}{2}} L\right)\left\{\int_{R}^{\infty} u_{n}^{2} d r\right\}^{\frac{1}{2}}\left\{\int_{R}^{\infty}\left[u_{n}(r)-u(r)\right]^{2} d r\right\}^{\frac{1}{2}} \\
\leq & \frac{1}{|\lambda|} \sup _{r \geq R} f\left(r, R^{\frac{1-N}{2}} L\right)\left\|u_{n}\right\|_{\lambda}\left\|u_{n}-u\right\|_{\lambda} \\
\leq & \frac{2 K^{2}}{|\lambda|} \sup _{r \geq R} f\left(r, R^{\frac{1-N}{2}} L\right), \quad \text { where } \quad L=(\min \{1,|\lambda|\})^{-\frac{1}{2}} K .
\end{aligned}
$$

Given $\varepsilon>0$, it follows from $(H 1)$ that there is $R_{\varepsilon}>0$ such that

$$
0 \leq \frac{2 K^{2}}{|\lambda|} \sup _{r \geq R_{\varepsilon}} f\left(r, R_{\varepsilon}^{\frac{1-N}{2}} L\right) \leq \varepsilon .
$$

But $H^{1}\left(0, R_{\varepsilon}\right)$ is compactly embedded in $L^{2}\left(0, R_{\varepsilon}\right)$ and so

$$
\int_{0}^{R_{\varepsilon}}\left[u_{n}(r)-u(r)\right]^{2} d r \stackrel{n}{\longrightarrow} 0
$$

Thus we see that

$$
\limsup _{n \rightarrow \infty}\left|\|u\|_{\lambda}^{2}-\left\|u_{n}\right\|_{\lambda}^{2}\right| \leq \varepsilon \quad \text { for any } \quad \varepsilon>0
$$

and conseqently $\left\|u_{n}\right\|_{\lambda}=\left\|u_{n}\right\|_{\lambda}$. Since $u_{n} \stackrel{n}{\rightarrow} u$ weakly in $H$ this implies that $\left\|u-u_{n}\right\|_{\lambda} \longrightarrow 0$ as $n \rightarrow \infty$ as required.

(d): Choose any $c>0$. To prove that the condition (d) is satisfied at the arbitrary value of $c$ we have chosen, let

$$
m_{n}=\inf \left\{\left\|J^{\prime}(u)\right\|\|u\|_{\lambda}: u \in A_{n}\right\}
$$

where

$$
A_{n}=\left\{u \in H: c-\frac{1}{n} \leq J(u) \leq c+\frac{1}{n} \text { and } \quad\|u\|_{\lambda} \geq n\right\}
$$


with $m_{n}=\infty$ if $A_{n}=\emptyset$.

Clearly (d) is satisfied if $m_{n}>0$ for some $n \in I N$. We now suppose that $m_{n}=0$ for all $n \in \mathbb{I}$ and show that this leads to a contradiction.

Indeed, if $m_{n}=0$ then there exists $u_{n} \in A_{n}$ such that

$$
\left\|J^{\prime}\left(u_{n}\right)\right\|\left\|u_{n}\right\|_{\lambda}<\frac{1}{n}
$$

and hence, if $m_{n}=0$ for all $n \in \mathbb{N}$, there is a sequence $\left\{u_{n}\right\} \in H$ such that

$$
J\left(u_{n}\right) \longrightarrow c, \quad\left\|u_{n}\right\|_{\lambda} \longrightarrow \infty \quad \text { and } \quad\left\|J^{\prime}\left(u_{n}\right)\right\|\left\|u_{n}\right\|_{\lambda}<\frac{1}{n} \quad \text { as } \quad n \rightarrow \infty .
$$

Thus $J^{\prime}\left(u_{n}\right) u_{n} \longrightarrow 0$ as $n \rightarrow \infty$ and we have

$$
\begin{gathered}
\frac{1}{2}\left\|u_{n}\right\|_{\lambda}^{2}-\int_{0}^{\infty} F_{1}\left(r, u_{n}(r)\right) d r=c+\circ(1) \\
\left\|u_{n}\right\|_{\lambda}^{2}-\int_{0}^{\infty} f\left(r, r^{\frac{1-N}{2}} u_{n}^{+}(r)\right) u_{n}(r)^{2} d r=\circ(1)
\end{gathered}
$$

where we again use o(1) to denote any quantity which tends to zero as $n \rightarrow \infty$. In fact we even have

$$
-\frac{1}{n}<J^{\prime}\left(u_{n}\right) u_{n}=\left\|u_{n}\right\|_{\lambda}^{2}-\int_{0}^{\infty} f\left(r, r^{\frac{1-N}{2}} u_{n}^{+}(r)\right) u_{n}(r)^{2} d r<\frac{1}{n}
$$

for all $n$. We claim that for any $t>0$ and $n \in I N$,

$$
J\left(t u_{n}\right) \leq \frac{t^{2}}{2 n}+\int_{0}^{\infty} \frac{1}{2} f\left(r, r^{\frac{1-N}{2}} u_{n}^{+}(r)\right) u_{n}(r)^{2}-F_{1}\left(r, u_{n}(r)\right) d r
$$

Indeed, for any $t>0$, at fixed $r>0$ and $n \in \mathbb{N}$, we set

$$
h(t)=\frac{1}{2} t^{2} f\left(r, r^{\frac{1-N}{2}} u_{n}^{+}(r)\right) u_{n}(r)^{2}-F_{1}\left(r, t u_{n}(r)\right)
$$

and observe that

$$
\begin{aligned}
h^{\prime}(t)= & t f\left(r, r^{\frac{1-N}{2}} u_{n}^{+}(r)\right) u_{n}(r)^{2}-f\left(r, r^{\frac{1-N}{2}} t u_{n}^{+}(r)\right) t u_{n}(r)^{2} \\
= & t u_{n}(r)^{2}\left\{f\left(r, r^{\frac{1-N}{2}} u_{n}^{+}(r)\right)-f\left(r, r^{\frac{1-N}{2}} t u_{n}^{+}(r)\right)\right\} \\
& \begin{cases}\geq 0 & \text { for } \quad 0<t \leq 1 \quad \text { by } \quad(H 2) . \\
\leq 0 & \text { for } \quad t \geq 1 .\end{cases}
\end{aligned}
$$

Hence,

$$
h(t) \leq h(1) \text { for all } t>0
$$


Now,

$$
\begin{aligned}
J\left(t u_{n}\right) & =\frac{1}{2} t^{2}\left\|u_{n}\right\|_{\lambda}^{2}-\int_{0}^{\infty} F_{1}\left(r, t u_{n}(r)\right) d r \\
& <\frac{1}{2} t^{2}\left\{\frac{1}{n}+\int_{0}^{\infty} f\left(r, r^{\frac{1-N}{2}} u_{n}^{+}(r)\right) u_{n}(r)^{2} d r\right\}-\int_{0}^{\infty} F_{1}\left(r, t u_{n}(r)\right) d r, \text { by }(3.16) . \\
& \leq \frac{t^{2}}{2 n}+\int_{0}^{\infty}\left\{\frac{1}{2} t^{2} f\left(r, r^{\frac{1-N}{2}} u_{n}^{+}(r)\right) u_{n}(r)^{2}-F_{1}\left(r, t u_{n}(r)\right)\right\} d r \\
& \leq \frac{t^{2}}{2 n}+\int_{0}^{\infty}\left\{\frac{1}{2} f\left(r, r^{\frac{1-N}{2}} u_{n}^{+}(r)\right) u_{n}(r)^{2}-F_{1}\left(r, u_{n}(r)\right)\right\} d r, \text { by }(3.18)
\end{aligned}
$$

This justifies the claim (3.17).

But,

$$
\begin{aligned}
J\left(u_{n}\right) & =\frac{1}{2}\left\|u_{n}\right\|_{\lambda}^{2}-\int_{0}^{\infty} F_{1}\left(r, u_{n}(r)\right) d r \\
& \geq \frac{1}{2}\left\{-\frac{1}{n}+\int_{0}^{\infty} f\left(r, r^{\frac{1-N}{2}} u_{n}^{+}(r)\right) u_{n}(r)^{2} d r\right\}-\int_{0}^{\infty} F_{1}\left(r, u_{n}(r)\right) d r
\end{aligned}
$$

so that

$$
\int_{0}^{\infty}\left\{\frac{1}{2} f\left(r, r^{\frac{1-N}{2}} u_{n}^{+}(r)\right) u_{n}(r)^{2}-F_{1}\left(r, u_{n}(r)\right)\right\} d r \leq \frac{1}{2 n}+J\left(u_{n}\right) .
$$

Combining (3.17) and (3.19) we find that

$$
J\left(t u_{n}\right) \leq \frac{\left(1+t^{2}\right)}{2 n}+J\left(u_{n}\right) \text { for all } t>0 \text { and } n \in I N
$$

Let

$$
w_{n}=\frac{2 \sqrt{c} u_{n}}{\left\|u_{n}\right\|_{\lambda}} .
$$

Clearly $\left\{w_{n}\right\}$ is bounded in $H$ and so we may pass to a subsequence and suppose that

$$
w_{n} \stackrel{n}{\rightarrow} w \text { weakly in } H
$$

We now claim that

$$
w \not \equiv 0 \text {. }
$$

In fact, if $w \equiv 0$, then $w_{n} \stackrel{n}{\rightarrow} 0$ uniformly on compact subsets of $(0, \infty)$. Hence, for any $K>0$,

$$
\begin{aligned}
0 & \leq \int_{0}^{\infty} F_{1}\left(r, w_{n}(r)\right) d r \\
& \leq \frac{1}{2} \int_{0}^{\infty} f\left(r, r^{\frac{1-N}{2}} w_{n}^{+}(r)\right) w_{n}(r)^{2} d r \\
& \leq \frac{1}{2} \int_{0}^{K} g(r) w_{n}^{2} d r+\frac{1}{2} \sup _{r \geq K} f\left(r, r^{\frac{1-N}{2}} w_{n}^{+}(r)\right) \int_{K}^{\infty} w_{n}(r)^{2} d r
\end{aligned}
$$


Since $\left\{u_{n}\right\}$ is bounded in $H$, there is a constant $L>0$ such that

$$
\left|w_{n}\right|_{2} \leq L \text { and }\left|w_{n}\right|_{\infty} \leq L \text { for all } n \in \mathbb{N}
$$

Hence,

$$
\sup _{r \geq K} f\left(r, r^{\frac{1-N}{2}} w_{n}^{+}(r)\right) \leq \sup _{r \geq K} f\left(r, K^{\frac{1-N}{2}} L\right) .
$$

By (H1), for any given $\varepsilon>0$, there is a $K_{\varepsilon}$ such that

$$
0 \leq f\left(r, K_{\varepsilon}^{\frac{1-N}{2}} L\right)<\varepsilon \text { for all } r \geq K_{\varepsilon} .
$$

Therefore

$$
0 \leq \int_{0}^{\infty} F_{1}\left(r, w_{n}(r)\right) d r \leq \frac{1}{2}|g|_{\infty} \int_{o}^{K_{\varepsilon}} w_{n}(r)^{2} d r+\frac{1}{2} L^{2} \varepsilon .
$$

Since $w_{n} \stackrel{n}{\rightarrow} 0$ uniformly on $\left[0, K_{\varepsilon}\right]$, it follows easily that

$$
\lim _{n \rightarrow \infty} \int_{0}^{\infty} F_{1}\left(r, w_{n}(r)\right) d r=0
$$

and consequently that

$$
J\left(w_{n}\right)=\frac{1}{2}\left\|w_{n}\right\|_{\lambda}^{2}+o(1)=2 c+o(1)
$$

where we again denote by $o(1)$ any quantity which tends to zero as $n \rightarrow \infty$.

$$
\begin{gathered}
\text { Setting } t_{n}=\frac{2 \sqrt{c}}{\left\|u_{n}\right\|_{\lambda}} \text {, we observe that } t_{n} \rightarrow 0 \text { as } n \rightarrow \infty \text { and that } \\
J\left(w_{n}\right)=J\left(t_{n} u_{n}\right) \leq \frac{1+t_{n}^{2}}{2 n}+J\left(u_{n}\right) \text {, by }(3.20) .
\end{gathered}
$$

But $J\left(u_{n}\right) \stackrel{n}{\longrightarrow} c$ and so we see that $(3.22)$ and (3.23) are contradictory since $c>0$. This justifies the claim (3.21), i.e. $w \not \equiv 0$.

The next step is to show that $w$ satisfies the following identity

$$
\int_{0}^{\infty} w^{\prime} \varphi^{\prime}-\lambda w \varphi+\frac{(N-1)(N-3)}{4 r^{2}} w \varphi-g(r) w^{+} \varphi d r=0
$$

for all $\varphi \in H$.

Set $p_{n}(r)=f\left(r, r^{\frac{1-N}{2}} u_{n}^{+}(r)\right)$ for $r>0$. We know that $p_{n}(r) \in C((0, \infty))$ with

$$
0 \leq p_{n}(r) \leq g(r), \forall r>0 .
$$


Passing to a suitable (diagonal) subsequence we can suppose that there is a function $h \in L_{\text {loc }}^{2}((0, \infty))$ such that

$$
p_{n} \stackrel{n}{\rightarrow} h \quad \text { weakly in } \quad L^{2}[a, b]
$$

and

$$
0 \leq h(r) \leq g(r) \text { a.e. on }(0, \infty)
$$

for every compact interval $[a, b] \subset(0, \infty)$.

Note that $h(r)=0$ whenever $w(r)<0$ and $h(r)=g(r)$ whenever $w(r)>0$.

Let $\varphi \in C_{0}^{\infty}((0, \infty))$ and let $[a, b]=\operatorname{supp} \varphi$. Since $w_{n} \stackrel{n}{\rightarrow} w$ uniformly on $[a, b]$, it follows that

$$
\int_{0}^{\infty} p_{n}(r) w_{n}(r) \varphi(r) d r \stackrel{n}{\longrightarrow} \int_{0}^{\infty} h(r) w^{+}(r) \varphi(r) d r .
$$

Since $\left\{p_{n} w_{n}\right\}$ is a bounded sequence in $L^{2}(0, \infty)$, we conclude that

$$
p_{n} w_{n} \stackrel{n}{\longrightarrow} h w^{+} \text {weakly in } L^{2}(0, \infty) \text {. }
$$

But

$$
\left|J^{\prime}\left(u_{n}\right) \varphi\right| \leq\left\|J^{\prime}\left(u_{n}\right)\right\|\|\varphi\|_{\lambda} \leq \frac{\|\varphi\|_{\lambda}}{n\left\|u_{n}\right\|_{\lambda}}
$$

and

$$
\begin{gathered}
\left|\int_{0}^{\infty} w_{n}^{\prime} \varphi^{\prime}-\lambda w_{n} \varphi+\frac{(N-1)(N-3)}{4 r^{2}} w_{n} \varphi-p_{n}(r) w_{n}^{+} \varphi d r\right| \\
=t_{n}\left|J^{\prime}\left(u_{n}\right) \varphi\right| \leq \frac{2 \sqrt{c}\|\varphi\|_{\lambda}}{n\left\|u_{n}\right\|_{\lambda}^{2}} .
\end{gathered}
$$

Recalling that $\left\|u_{n}\right\|_{\lambda} \stackrel{n}{\rightarrow} \infty$ and that $w_{n} \stackrel{n}{\rightarrow} w$ weakly in $H$, this shows that

$$
\int_{0}^{\infty} w^{\prime} \varphi^{\prime}-\lambda w \varphi+\frac{(N-1)(N-3)}{4 r^{2}} w \varphi-h(r) w^{+} \varphi d r=0
$$

for all $\varphi \in C_{0}^{\infty}((0, \infty))$, and then, by approximation, for all $\varphi \in H$.

Setting $\varphi=w^{-}$, this yields $\left\|w^{-}\right\|_{\lambda}^{2}=0$ and so we have that $w \equiv w^{+} \geq 0$ on $(0, \infty)$. But then the maximum principle implies that $w(r)>0$ on $(0, \infty)$. Thus, we have that $w \in H, w(r)>0$ on $(0, \infty)$ and

$$
\int_{0}^{\infty} w^{\prime} \varphi^{\prime}-\lambda w \varphi+\frac{(N-1)(N-3)}{4 r^{2}} w \varphi-g(r) w \varphi d r=0
$$

for all $\varphi \in H$.

Setting $z(x)=|x|^{\frac{1-N}{2}} w(|x|)$, as in Lemma 3.1 and Theorem 3.2, we find that $z \in H^{1}\left(\mathbb{R}^{N}\right)$ and

$$
\int_{0}^{\infty} \nabla z \cdot \nabla v d x=\int_{0}^{\infty}\{\lambda+g(r)\} z v d x, \forall v \in C_{0}^{\infty}\left(\mathbb{R}^{N}\right)
$$


This implies that $z \in H^{2}\left(\mathbb{R}^{N}\right)$ with $z(x)>0$ for $|x| \neq 0$ and

$$
S z=\lambda z, \text { where } S z=-\triangle z-g(|x|) z .
$$

If $\Lambda<-g(\infty)$, we know that, by Lemma 3.5(b), there exists $\varphi \in H^{2}$ such that $S \varphi=\Lambda \varphi$ and $\varphi(x)>0$ on $\mathbb{R}^{N}$. Then

$$
\lambda \int_{\mathbb{R}^{N}} z \varphi d x=\int_{\mathbb{R}^{N}}(S z) \varphi d x=\int_{\mathbb{R}^{N}} z(S \varphi) d x=\Lambda \int_{\mathbb{R}^{N}} z \varphi d x
$$

So that $\lambda=\Lambda$, which is false.

The only other possibility is that $\Lambda=-g(\infty)$ and in this case, since $\lambda>\Lambda$, there exists $R>0$ such that

$$
\lambda+g(r)-\frac{(N-1)(N-3)}{r^{2}} \geq \frac{\lambda-\Lambda}{2}, \text { for all } r \geq R
$$

Let $I$ denote the interval $\left(R, R+\frac{2 \pi}{\sqrt{\lambda-\Lambda}}\right)$ and set

$$
\varphi(r)= \begin{cases}\sin \frac{\sqrt{\lambda-\Lambda}}{2}(r-R), & \text { for } r \in I \\ 0, & \text { for all } r \notin I .\end{cases}
$$

Then $\varphi \geq 0$ on $(0, \infty), \varphi>0$ on $I$ and $\varphi \in H$. Using (3.25) we find that

$$
\int_{I} w^{\prime} \varphi^{\prime} d r=\int_{I}\left\{\lambda+g(r)-\frac{(N-1)(N-3)}{r^{2}}\right\} w \varphi d r \geq \frac{\lambda-\Lambda}{2} \int_{I} w \varphi d r .
$$

But

$$
\int_{I} w^{\prime} \varphi^{\prime} d r=-\int_{I} w \varphi^{\prime \prime} d r=\frac{\lambda-\Lambda}{4} \int_{I} w \varphi d r
$$

Since $\int_{I} w \varphi d r>0$, we again have a contradiction.

Thus we see that the assumption that $m_{n}=0$ for all $n \in I N$ always leads to a contradiction. This proves that $(\mathbf{d})$ is satisfied and the proof is complete.\#

\section{ACKNOWLEDGEMENT}

This work was supported by Swiss National Science Foundation (Project number $20-46937.96)$. The second author would like to thank the Swiss NSF for supporting his stay at the EPFL and also the Department of Mathematics at the EPFL for its hospitality. 


\section{References}

[1] P. Bartolo, V. Benci \& D. Fortunato, Abstract critical point theorems and applications to some nonlinear problems with strong resonance at infinity, Nonlin. Anal. TMA, 7(1983)9, 981-1012.

[2] F.A. Berezin \& M.A. Shubin, The Schrödinger Equation, Kluwer, Dordrecht, 1991.

[3] R. Dautray \& J.L. Lions, Analyse Mathématique et Calcul Numérique pour les Sciences et les Techniques, Vol. 1, Masson, Paris, 1984.

[4] G.B. Folland, Introduction to Partial Differential Equations, Second edition, Princeton University Press, 1995.

[5] D. Gilbarg \& N.S. Trudinger, Elliptic Partial Differential Equations of Second Order, Springer, Berlin-New York, 1983.

[6] L. Jeanjean, On the existence of bounded Palais-Smale sequences and application to a Landesman-Lazer type problem set on $R^{N}$, preprint

[7] P.H. Rabinowitz, Minimax Methods in Critical Point Theory with Applications to Differential Equations, CBMS Reg. Conf. Ser. in Math. No. 65, Amer. Math. Soc., Providence, R.I., 1986.

[8] E. Stein, Singular Integrals and Differentiability Properties of Functions, Princeton Univ. Press, Princeton, NJ, 1970.

[9] C.A. Stuart \& H.S. Zhou, A variational problem related to self-trapping of an electromagnetic field, Math. Methods in Applied Sci., 19(1996), 1397-1407.

[10] M. Willem, Minimax Theorems, Birkäuser, Basel, 1996.

[11] H.S. Zhou, Positive solution for a semilinear elliptic equation which is almost linear at infinity, to appear in J. Applied Math. Physics (ZAMP). 